\title{
Triphosgene mediated chlorination of Baylis-Hillman adducts
}

\author{
NARENDER REDDY THATIKONDA, NAGA SESHA SAI PAVAN KUMAR CHEBOLU, \\ MAHENDAR BUDDE and JAYATHIRTHA RAO VAIDYA* \\ Organic Chemistry Division II, Indian Institute of Chemical Technology, Uppal Road, \\ Tarnaka, Hyderabad 500 607, India \\ e-mail: jrao@iict.res.in
}

MS received 22 August 2011; revised 19 November 2011; accepted 25 November 2011

\begin{abstract}
An efficient method for the preparation of allyl chlorides from Baylis-Hillman adducts has been developed using triphosgene/pyridine system. This method is best illustrated by its advantages like operational simplicity, excellent yields, short reaction time, simple procedure and stereoselectivity.
\end{abstract}

Keywords. Baylis-Hillman adducts; triphosgene; chlorination; stereoselectivity; simple procedure.

\section{Introduction}

Baylis-Hillman reaction ${ }^{1}$ is now a standard $\mathrm{C}-\mathrm{C}$ bond formation reaction widely used in synthetic organic chemistry. The reaction has been extensively studied and various applications are reported in the literature. ${ }^{2}$ The derivatives of Baylis-Hillman adducts, in particular, Baylis-Hillman allyl halides have been used as valuable intermediates in the preparation of several heterocyclic compounds and also in stereo-selective processes. ${ }^{3}$ Chlorination of BaylisHillman adducts using various reagents/protocols are reported in the literature viz., $\mathrm{HCl} / \mathrm{H}_{2} \mathrm{SO}_{4},{ }^{4} \mathrm{FeCl}_{3}$ or $\mathrm{InCl}_{3},{ }^{5} \mathrm{PPh}_{3} / \mathrm{CCl}_{4},{ }^{6} \mathrm{PPh}_{3} / \mathrm{Cl}_{3} \mathrm{CCONH}_{2},{ }^{7} \mathrm{TCT} / \mathrm{DMF},{ }^{8}$ $(\mathrm{COCl})_{2} / \mathrm{DMF},{ }^{9} \mathrm{DMF} / \mathrm{POCl}_{3},{ }^{10} \mathrm{NEt}_{3} / \mathrm{MsCl},{ }^{11}$ and also using ionic liquid as medium. ${ }^{12}$ Unfortunately, many of these suffer from limitations such as harsh reaction conditions, lack of general applicability, longer reaction times, and only one report ${ }^{6}$ is available in the literature for the conversion of nitrile containing Baylis-Hillman adducts to their allyl chlorides. Therefore, reinvestigation of the classical conditions seemed warranted for developing suitable conditions for the synthesis of allyl chlorides. Triphosgene [bis(trichloromethyl)carbonate] is yet another important chlorinating agent has been found to be an excellent activating agent in several organic reactions. ${ }^{13}$ The efficiency of triphosgene (white crystalline compound) as a synthetic auxiliary, as a carbonylating agent, as a chlorinating agent, as a replacement of $\mathrm{POCl}_{3}$ in the preparation of many important classes of organic compounds has been investigated in the last twenty years. ${ }^{14}$ In continuation of our

*For correspondence interest on the chemical transformations using BaylisHillman chemistry, ${ }^{15}$ we report here a new and convenient method for the chlorination of Baylis-Hillman adducts to obtain the allyl chlorides in a stereoselective manner. In this paper, we describe the use of triphosgene as an excellent reagent for the easy and mild conversion of Baylis-Hillman adducts into their allyl chlorides. The chlorination using triphosgene is fast, smooth, clean and high yielding.

\section{Experimental}

\subsection{General}

All the chemicals used were of reagent grade obtained from local suppliers, Aldrich and Fluka. All reactions were performed in oven-dried glassware under nitrogen atmosphere. Analytical thin layer chromatography (TLC) was performed on silica gel plates and TLC visualization was carried out with UV. Melting points were determined on a Mettlers-Temp and are uncorrected. IR Spectra were recorded using a Perkin-Elmer-1600 FT-IR spectrometer; $v$ in $\mathrm{cm}^{-1} .{ }^{1} \mathrm{H}$ and ${ }^{13} \mathrm{C}$-NMR spectrum $\left(\mathrm{CDCl}_{3} / \mathrm{DMSO}-\mathrm{d}_{6}\right)$ was recorded with Gemini200 and Bruker-Avance-300 instruments; chemical shifts $\delta$ in ppm relative to $\mathrm{SiMe}_{4}$ as an internal standard, couplings in Hz. HRMS (ESI) data were recorded on a QSTAR XL High resolution mass spectrometer; in $\mathrm{m} / z$ (rel. \%). GC was recorded on GC-17A Gas Chromatograph SHIMADZU system (column: Zebron-1 C49045 $30 \mathrm{~m} \times 53 \mathrm{~mm}$ I.D. $\times 1.5 \mu \mathrm{F}$. T.), Oven program $50^{\circ} \mathrm{C}$ for $5 \mathrm{~min}$ temp. raised $10^{\circ} \mathrm{C} / \mathrm{min}$ to $280^{\circ} \mathrm{C}$; hold $5 \mathrm{~min}$; gas flow rate $1 \mathrm{~mL} / \mathrm{min}$. 
2.2 General experimental procedure for the synthesis of allyl chlorides from Baylis-Hillman adducts (1a-v)

To a stirred solution of triphosgene $(0.93 \mathrm{~g}, 3.14 \mathrm{mmol})$ in dichloromethane $(5 \mathrm{~mL})$ at $0^{\circ} \mathrm{C}$, pyridine $(1.26 \mathrm{~mL}$, $15.72 \mathrm{mmol}$ ) was added drop-wise followed by BaylisHillman adduct $(\mathbf{1 m})(0.5 \mathrm{~g}, 3.14 \mathrm{mmol})$ in DCM $(5 \mathrm{~mL})$ and stirred the reaction at room temperature (Monitored by TLC). After completion of the reaction, the reaction mixture was diluted with DCM $(10 \mathrm{~mL})$; the organic layer was washed with water $(5 \mathrm{~mL})$, sat. $\mathrm{CuSO}_{4}$ solution $(5 \mathrm{~mL})$, dried over $\mathrm{Na}_{2} \mathrm{SO}_{4}$ and the solvent was removed under reduced pressure to get the desired allyl chloride $(\mathbf{2 m})$ as colourless liquid $(0.57 \mathrm{~g}$, 95\%) $R_{f}=0.8$ (20\% EtOAc/hexane). Same experimental procedure was adopted for the preparation of other allyl chlorides.

2.2a (Z)-Methyl 2-(chloromethyl)-3-phenylacrylate (2a): Colourless liquid; Yield 95\%; ${ }^{1} \mathrm{H}$ NMR $(300 \mathrm{MHz}$, $\left.\mathrm{CDCl}_{3}\right): \delta=7.83(s, 1 \mathrm{H}), 7.52-7.43(\mathrm{~m}, 2 \mathrm{H}), 7.45-$ $7.25(m, 3 \mathrm{H}), 4.43(s, 2 \mathrm{H}), 3.87(s, 3 \mathrm{H})$; IR (Neat): 2988, 2853, 1750, 1630, 1250,1170 $\mathrm{cm}^{-1}$; MS (EI): $m / z 210\left[\mathrm{M}^{+}\right], 176$ (100), 131, 115; GC: $Z: E=97: 3$.

$2.2 \mathrm{~b}$ (Z)-Methyl 2-(chloromethyl)-3-(4-chlorophenyl) acrylate (2b): Colourless liquid; Yield 92\%; ${ }^{1} \mathrm{H}$ NMR $\left(300 \mathrm{MHz}, \mathrm{CDCl}_{3}\right): \delta=7.71(s, 1 \mathrm{H}), 7.49(d, J=$ $8.8 \mathrm{~Hz}, 2 \mathrm{H}), 7.42(d, J=7.8 \mathrm{~Hz}, 2 \mathrm{H}), 4.39(s, 2 \mathrm{H})$, 3.87 ( $s, 3 \mathrm{H})$; IR (Neat): 2953, 2849, 1718, 1633, 1599, 1509, 1439, 1278, 1230, $1163 \mathrm{~cm}^{-1}$; MS (EI): $m / z 244$ $\left[\mathrm{M}^{+}\right.$], 209, 149 (100), 130, 115; GC: $Z: E=95: 5$.

2.2c (Z)-Methyl 2-(chloromethyl)-3-(4-ethylphenyl) acrylate (2c): Colourless liquid; Yield 95\%; ${ }^{1} \mathrm{H}$ NMR $\left(300 \mathrm{MHz}, \mathrm{CDCl}_{3}\right): \delta=7.80(s, 1 \mathrm{H}), 7.40(d, 2 \mathrm{H}$, $J=7.9 \mathrm{~Hz}), 7.26(d, 2 \mathrm{H}, J=7.9 \mathrm{~Hz}), 4.45(s, 2 \mathrm{H})$, $3.86(s, 3 \mathrm{H}), 2.72(q, 2 \mathrm{H}, J=7.5 \mathrm{~Hz}), 1.29(t, 3 \mathrm{H}$, $J=7.5 \mathrm{~Hz}) ;{ }^{13} \mathrm{C} \mathrm{NMR}\left(75 \mathrm{MHz}, \mathrm{CDCl}_{3}\right): \delta=166.8$, $146.4,143.9,131.4,129.8,128.8,128.4,52.3,39.3$, 28.7, 15.2; IR (Neat): 2965, 1718, 1628, 1509, 1436, $1272 \mathrm{~cm}^{-1} ; \mathrm{MS}$ (ESI): $m / z 261[\mathrm{M}+\mathrm{Na}]^{+}$; HRMS (ESI) calcd. for $\mathrm{C}_{13} \mathrm{H}_{15} \mathrm{ClO}_{2} \mathrm{Na}$ : 261.0658; found: 261.0653; GC: $Z: E=96: 4$.

2.2d (Z)-Methyl 2-(chloromethyl)-3-(4-isopropylphenyl) acrylate (2d): Colourless liquid; Yield 93\%; ${ }^{1} \mathrm{H}$ NMR $\left(300 \mathrm{MHz}, \mathrm{CDCl}_{3}\right): \delta=7.79(s, 1 \mathrm{H}), 7.47(d, J=$ $7.9 \mathrm{~Hz}, 2 \mathrm{H}), 7.28(s, J=8.9 \mathrm{~Hz}, 2 \mathrm{H}), 4.44(s, 2 \mathrm{H})$, $3.85(s, 3 \mathrm{H}), 2.94(m, J=6.9 \mathrm{~Hz}, 1 \mathrm{H}), 1.27$ $(d, J=6.9 \mathrm{~Hz}, 6 \mathrm{H}) ;{ }^{13} \mathrm{C} \mathrm{NMR}\left(75 \mathrm{MHz}, \mathrm{CDCl}_{3}\right)$ : $\delta=166.8,150.9,143.8,129.9,128.9,126.9,126.6$, 126.2, 52.3, 39.3, 34.0, 23.7. IR (Neat): 2961, 1717, 1628, 1509, 1436, $1281 \mathrm{~cm}^{-1}$; MS (ESI): $\mathrm{m} / z 275$ $[\mathrm{M}+\mathrm{Na}]^{+}$; HRMS (ESI) calcd. for $\mathrm{C}_{14} \mathrm{H}_{17} \mathrm{ClO}_{2} \mathrm{Na}$ : 275.0814; found: 275.0826; GC: $Z: E=96: 4$.

2.2e (Z)-Methyl 2-(chloromethyl)-3-(4-methoxyphenyl) acrylate (2e): Colourless liquid; Yield 90\%; ${ }^{1} \mathrm{H}$ NMR $\left(300 \mathrm{MHz}, \mathrm{CDCl}_{3}\right): \delta=7.77(s, 1 \mathrm{H}), 7.54(d, J=$ $8.3 \mathrm{~Hz}, 2 \mathrm{H}), 6.95(d, J=9.0 \mathrm{~Hz}, 2 \mathrm{H}), 4.47(s, 2 \mathrm{H})$, $3.86(s, 3 \mathrm{H}), 3.84(s, 3 \mathrm{H})$; IR (Neat): 2960, 2840, 1908, $1718,1633,1599,1439,1278 \mathrm{~cm}^{-1}$; MS (EI): $m / z 240$ $\left[\mathrm{M}^{+}\right], 205,145$ (100) 131, 115; GC: $Z: E=95: 5$.

$2.2 \mathrm{f}$ (Z)-Methyl 2-(chloromethyl)-3-(4-fluorophenyl) acrylate (2f): Colourless liquid; Yield 93\%; ${ }^{1} \mathrm{H}$ NMR $\left(300 \mathrm{MHz}, \mathrm{CDCl}_{3}\right): \delta=7.78(s, 1 \mathrm{H}), 7.56(t, J=$ $8.8 \mathrm{~Hz}, J=7.8 \mathrm{~Hz}, 2 \mathrm{H}), 7.15(t, J=8.8 \mathrm{~Hz}, 2 \mathrm{H})$, $4.40(s, 2 \mathrm{H}), 3.80(s, 3 \mathrm{H})$; IR (Neat): 2952, 1718, 1631, 1490, 1438, 1312, 1281, 1208, $1090 \mathrm{~cm}^{-1}$; MS (EI): $m / z 228\left[\mathrm{M}^{+}\right], 193,133$ (100), 115, 107; GC: $Z: E=98: 2$.

$2.2 \mathrm{~g}$ (Z)-Methyl 2-(chloromethyl)-3-(2-chlorophenyl) acrylate (2g): Colourless liquid; Yield 90\%; ${ }^{1} \mathrm{H}$ NMR $\left(300 \mathrm{MHz}, \mathrm{CDCl}_{3}\right): \delta=7.92(\mathrm{~s}, 1 \mathrm{H}), 7.69-7.65(\mathrm{~m}$, $1 \mathrm{H}), 7.46-7.32(m, 3 \mathrm{H}), 4.32(s, 2 \mathrm{H}), 3.90(s, 3 \mathrm{H})$; IR (Neat): 2982, 1717, 1636, 1469, 1439, 1371, 1288, 1208, $1179 \mathrm{~cm}^{-1}$; MS (EI): $m / z 244\left[\mathrm{M}^{+}\right], 209,149$ (100), 130, 115; GC: $Z: E=98: 2$.

$2.2 \mathrm{~h}$ (Z)-Ethyl 2-(chloromethyl)-3-(4-chlorophenyl) acrylate (2h): Colourless solid; Yield 92\%; M.p.: $78-80^{\circ} \mathrm{C} ;{ }^{1} \mathrm{H}$ NMR $\left(300 \mathrm{MHz}, \mathrm{CDCl}_{3}\right): \delta=7.76(s$, $1 \mathrm{H}), 7.50(d, J=8.30 \mathrm{~Hz}, 2 \mathrm{H}), 7.47(d, J=8.6 \mathrm{~Hz}$, $2 \mathrm{H}), 4.39(s, 2 \mathrm{H}), 4.36(q, J=7.1 \mathrm{~Hz}, 2 \mathrm{H}), 1.41$ $(t, J=7.1 \mathrm{~Hz}, 3 \mathrm{H})$; IR (KBr): 2924, 2853, 1701, 1624, 1489, 1378, 1263, 1213, $1179 \mathrm{~cm}^{-1}$; MS (EI): $258\left[\mathrm{M}^{+}\right], 223,195,159$ (100), 149, 131, 115; GC: $Z: E=97: 3$.

$2.2 \mathrm{i}$ (Z)-Ethyl 2-(chloromethyl)-3-(4-methoxyphenyl) acrylate (2i): Colourless liquid; Yield 90\%; ${ }^{1} \mathrm{H}$ NMR $\left(300 \mathrm{MHz}, \mathrm{CDCl}_{3}\right): \delta=7.78(s, 1 \mathrm{H}), 7.57(d, J=$ $8.3 \mathrm{~Hz}, 2 \mathrm{H}), 6.94(d, J=9.0 \mathrm{~Hz}, 2 \mathrm{H}), 4.49(s, 2 \mathrm{H})$, $4.36(q, J=7.1 \mathrm{~Hz}, 2 \mathrm{H}), 3.86(s, 3 \mathrm{H}), 1.39(t, J=$ $7.1 \mathrm{~Hz}, 3 \mathrm{H})$. IR (Neat): 2966, 2848, 1908, 1708, 1633, 1599, 1439, $1278 \mathrm{~cm}^{-1}$; MS (EI): $254\left[\mathrm{M}^{+}\right], 219$ (100) $145,131,115$. GC: $Z: E=98: 2$. 
$2.2 \mathrm{j}$ (Z)-Ethyl 2-(chloromethyl)-3-(2,4-dichlorophenyl) acrylate (2j): Colourless solid; Yield 90\%; M.p.: 74$76^{\circ} \mathrm{C} ;{ }^{1} \mathrm{H}$ NMR $\left(300 \mathrm{MHz}, \mathrm{CDCl}_{3}\right): \delta=7.82(s, 1 \mathrm{H})$, $7.63(d, J=7.8 \mathrm{~Hz}, 2 \mathrm{H}), 7.40(s, 1 \mathrm{H}), 7.36(d, J=$ $7.8 \mathrm{~Hz}, 2 \mathrm{H}), 4.35(q, J=6.8 \mathrm{~Hz}, 2 \mathrm{H}), 4.29(s, 2 \mathrm{H})$, $1.41(t, J=6.8 \mathrm{~Hz}, 3 \mathrm{H})$; IR (KBr): 2980, 2928, 1717 , $1583,1469,1375,1280,1251,1178 \mathrm{~cm}^{-1}$; MS (EI): $292\left[\mathrm{M}^{+}\right]$, 257, 247, 229, 193 (100), 149, 113; GC: $Z: E=98: 2$.

$2.2 \mathrm{k}$ (Z)-Ethyl 2-(chloromethyl)-3-(4-nitrophenyl) acrylate (2k): Yellow solid; Yield 93\%; M.p.: 77$79^{\circ} \mathrm{C} ;{ }^{1} \mathrm{H}$ NMR $\left(300 \mathrm{MHz}, \mathrm{CDCl}_{3}\right): \delta=8.33(d$, $J=9.0 \mathrm{~Hz}, 2 \mathrm{H}), 7.83(s, 1 \mathrm{H}), 7.72(d, J=9.0 \mathrm{~Hz}$, $2 \mathrm{H}), 4.39(q, J=6.7 \mathrm{~Hz}, 2 \mathrm{H}), 4.36(s, 2 \mathrm{H}), 1.43(t$, $J=6.7 \mathrm{~Hz}, 3 \mathrm{H})$; IR (KBr): 2920, 2855, 1595, 1510, 1438, 1344, 1258, $1105 \mathrm{~cm}^{-1}$; MS (EI): $269\left[\mathrm{M}^{+}\right], 234$, 150, 129, 115, 79 (100); GC: $Z: E=95: 5$.

2.21 (Z)-Ethyl 2-(chloromethyl)pent-2-enoate (2l): Colourless liquid; Yield 89\%; ${ }^{1} \mathrm{H}$ NMR $(300 \mathrm{MHz}$, $\left.\mathrm{CDCl}_{3}\right): \delta=6.96(t, J=7.7 \mathrm{~Hz}, 1 \mathrm{H}), 4.28(s, 2 \mathrm{H})$, $4.27(q, J=7.1 \mathrm{~Hz}, 2 \mathrm{H}), 2.40(q, J=7.5 \mathrm{~Hz}, 2 \mathrm{H})$, $1.35(t, J=7.1 \mathrm{~Hz}, 3 \mathrm{H}), 1.17(t, J=7.5 \mathrm{~Hz}, 3 \mathrm{H})$; IR (Neat): 2977, 2938, 1779, 1717, 1645, 1460, 1374, 1279, $1181 \mathrm{~cm}^{-1}$; MS (EI): $176\left[\mathrm{M}^{+}\right], 157,141,133$, 113, 99, 57 (100); GC: $Z: E=90: 10$.

2.2m (E)-2-(Chloromethyl)-3-phenylacrylonitrile (2m): Colourless liquid; Yield 95\%; ${ }^{1} \mathrm{H}$ NMR $(300 \mathrm{MHz}$, $\left.\mathrm{CDCl}_{3}\right): \delta=7.78-7.75(\mathrm{~m}, 2 \mathrm{H}), 7.44(t, 3 \mathrm{H}), 7.18$ $(s, 1 \mathrm{H}), 4.29$ ( $s, 2 \mathrm{H})$; IR (Neat): 2955, 2830, 2210, 1602, 1590, 1510, 1439, 1258, $1105 \mathrm{~cm}^{-1}$; MS (EI): $177\left[\mathrm{M}^{+}\right], 142,127,115$ (100), 102; GC: $Z: E=3: 97$.

2.2n (E)-2-(Chloromethyl)-3-(4-ethylphenyl) acrylonitrile (2n): Colourless liquid; Yield 95\%; ${ }^{1} \mathrm{H}$ NMR $\left(300 \mathrm{MHz}, \mathrm{CDCl}_{3}\right): \delta=7.71(d, J=8.1 \mathrm{~Hz}$, $2 \mathrm{H}), 7.26(d, J=8.1 \mathrm{~Hz}, 2 \mathrm{H}), 7.10(s, 1 \mathrm{H}), 4.28(s$, $2 \mathrm{H}), 2.70(q, J=7.5 \mathrm{~Hz}, 2 \mathrm{H}), 1.20(t, J=7.5 \mathrm{~Hz}$, $3 \mathrm{H}) ;{ }^{13} \mathrm{C}$ NMR $\left(75 \mathrm{MHz}, \mathrm{CDCl}_{3}\right): \delta=148.3,146.7$, $129.4,128.7,128.5,117.2,106.3,46.2,28.8,15.1$; IR (Neat): 2968, 2217, 1607, 1621, $1266 \mathrm{~cm}^{-1}$; MS (ESI): $m / z 228[\mathrm{M}+\mathrm{Na}]^{+}$. HRMS (ESI) calcd. for $\mathrm{C}_{12} \mathrm{H}_{12} \mathrm{ClNNa}$ : 228.0555; found: 228.0553; GC: $Z: E=5: 95$.

2.2o (E)-2-(Chloromethyl)-3-(3-fluorophenyl) acrylonitrile (2o): Colourless solid; Yield 88\%; M.p.: $72-74{ }^{\circ} \mathrm{C} ;{ }^{1} \mathrm{H}$ NMR $\left(300 \mathrm{MHz}, \mathrm{CDCl}_{3}\right): \delta=7.58(d$, $J=7.9 \mathrm{~Hz}, 2 \mathrm{H}), 7.48-7.41(m, 3 \mathrm{H}), 7.16(s, 1 \mathrm{H}), 4.29$ $(s, 2 \mathrm{H})$; IR (KBr): 2963, 2855, 2210, 1891, 1602, 1510, 1417, 1246, $1169 \mathrm{~cm}^{-1}$; MS (EI): $m / z 195\left[\mathrm{M}^{+}\right], 160$, 140, 133, 120; GC: $Z: E=10: 90$.

$2.2 \mathrm{p}$ (E)-3-(3-Bromophenyl)-2-(chloromethyl) acrylonitrile (2p): Colourless solid; Yield 85\%; M.p.: $76-78^{\circ} \mathrm{C} ;{ }^{1} \mathrm{H}$ NMR $\left(300 \mathrm{MHz}, \mathrm{CDCl}_{3}\right): \delta=7.82(d$, $J=9.0 \mathrm{~Hz}, 2 \mathrm{H}), 7.58-7.55(\mathrm{~m}, 1 \mathrm{H}), 7.36-7.30(t$, $J=8.3 \mathrm{~Hz}, 1 \mathrm{H}), 7.13(s, 1 \mathrm{H}), 4.29(s, 2 \mathrm{H})$; IR $(\mathrm{KBr})$ : 2963, 2925, 2214, 1314, 1467, 1268, $1215 \mathrm{~cm}^{-1}$; MS (EI): $m / z 254\left[\mathrm{M}^{+}\right], 220,141$ (100) 114; GC: $Z: E=4: 96$.

$2.2 \mathrm{q}$ (E)-2-(Chloromethyl)-3-(3-chlorophenyl) acrylonitrile (2q): Colourless solid; Yield 88\%; M.p.: $75-77^{\circ} \mathrm{C} ;{ }^{1} \mathrm{H} \mathrm{NMR}\left(300 \mathrm{MHz}, \mathrm{CDCl}_{3}\right): \delta=7.75-7.73$ $(m, 1 \mathrm{H}), 7.66(s, 1 \mathrm{H}), 7.41-7.39(m, 2 \mathrm{H}), 7.14(s, 1 \mathrm{H})$, 4.28 (s, 2H); IR (KBr): 2925, 2855, 2212, 1616, 1587 , 1486, 1407, 1266, $1090 \mathrm{~cm}^{-1}$; MS (EI): $m / z 212\left[\mathrm{M}^{+}\right.$], 176, 160, 140 (100), 133, 125, 113; GC: $Z: E=10: 90$.

2.2r (E)-2-(Chloromethyl)-3-(4-chlorophenyl) acrylonitrile (2r): Colourless solid; Yield 92\%; M.p. $73-75^{\circ} \mathrm{C}$; ${ }^{1} \mathrm{H}$ NMR $\left(300 \mathrm{MHz}, \mathrm{CDCl}_{3}\right): \delta=7.73(d$, $J=8.3 \mathrm{~Hz}, 2 \mathrm{H}), 7.43(d, J=8.3 \mathrm{~Hz}, 2 \mathrm{H}), 7.14(s$, 1H), 4.28 (s, 2H); IR (KBr): 2925, 2855, 2212, 1616, 1587, 1486, 1407, 1266, $1090 \mathrm{~cm}^{-1}$; MS (EI): $m / z 212$ $\left[\mathrm{M}^{+}\right], 176$ (100) 140, 113, 99; GC: Z:E=1:99.

2.2s (E)-3-(4-Bromophenyl)-2-(chloromethyl) acrylonitrile (2s): Colourless solid; Yield 93\%; M.p.: $72-74^{\circ} \mathrm{C} ;{ }^{1} \mathrm{H}$ NMR $\left(300 \mathrm{MHz}, \mathrm{CDCl}_{3}\right): \delta=7.66(d$, $J=8.3 \mathrm{~Hz}, 2 \mathrm{H}), 7.59(d, J=8.3 \mathrm{~Hz}, 2 \mathrm{H}), 7.13(s$, 1H), 4.27 (s, 2H); IR (KBr): 2924, 2855, 2211, 1616, 1582, 1481, 1403, 1261, $1071 \mathrm{~cm}^{-1}$; MS (EI): $m / z 254$ (100) $\left[\mathrm{M}^{+}\right], 220,140,114,100 ; \mathrm{GC}: Z: E=5: 95$.

2.2t (E)-2-(Chloromethyl)-3-(4-fluorophenyl) acrylonitrile (2t): Colourless solid; Yield 96\%; M.p.: $70-72{ }^{\circ} \mathrm{C} ;{ }^{1} \mathrm{H}$ NMR $\left(300 \mathrm{MHz}, \mathrm{CDCl}_{3}\right): \delta=7.98$

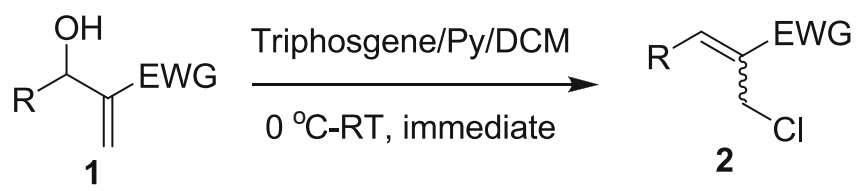

EWG $=$ COOEt, COOMe, CN

$\mathrm{R}=$ alkyl, aryl

Scheme 1. Chlorination of Baylis-Hillman adducts. 
Table 1. Comparison study of bases in chlorination of Baylis-Hillman adducts using triphosgene for the reference reaction $(\mathbf{1 m - 2 m})$.

\begin{tabular}{llcc}
\hline S. No & Base & Time & $\begin{array}{c}\text { Isolated } \\
\text { yield }(\%)^{*}\end{array}$ \\
\hline 1 & Pyridine & $10 \mathrm{~min}$ & 95 \\
2 & DMAP & $15 \mathrm{~min}$ & 90 \\
3 & DBU & $4 \mathrm{~h}$ & 85 \\
4 & DABCO & $12 \mathrm{~h}$ & 20 \\
5 & Piperidine & $12 \mathrm{~h}$ & 30 \\
6 & Diisopropyl Ethyl Amine & $12 \mathrm{~h}$ & - \\
7 & Et $_{3} \mathrm{~N}$ & $12 \mathrm{~h}$ & - \\
\hline
\end{tabular}

* Reaction was carried out at $0^{\circ} \mathrm{C}$ in $\mathrm{DCM}$ as solvent

$(t, J=8.8 \mathrm{~Hz}, 2 \mathrm{H}), 7.31(s, 1 \mathrm{H}), 7.30(d, J=8.8 \mathrm{~Hz}$, 2H), 4.45 ( $s, 2 \mathrm{H})$; IR (KBr): 2965, 2214, 1891, 1602, 1510, 1417, 1246, $1163 \mathrm{~cm}^{-1}$; MS (EI): $m / z 195\left[\mathrm{M}^{+}\right]$, 160 (100) 140, 133, 120,106; GC: $Z: E=2: 98$.

$2.2 \mathrm{u}$ (E)-2-(Chloromethyl)-3-(4-methoxyphenyl) acrylonitrile (2u): Colourless solid; Yield 93\%; M.p.: $71-73^{\circ} \mathrm{C} ;{ }^{1} \mathrm{H}$ NMR $\left(300 \mathrm{MHz}, \mathrm{CDCl}_{3}\right): \delta=7.77(d$, $J=8.8 \mathrm{~Hz}, 2 \mathrm{H}), 7.09(s, 1 \mathrm{H}), 6.93(d, J=8.8 \mathrm{~Hz}$, 2H), $4.27(s, 2 \mathrm{H}), 3.80(s, 3 \mathrm{H})$; IR (KBr): 2962, 2841, 2214, 1601, 1511, 1260, $1179 \mathrm{~cm}^{-1}$; MS (EI): $\mathrm{m} / \mathrm{z}$ $207\left[\mathrm{M}^{+}\right], 172$ (100), 157, 140, 128, 115, 102; GC: $Z: E=5: 95$.

$2.2 \mathrm{v}$ (E)-2-(Chloromethyl)-3-(4-nitrophenyl) acrylonitrile (2v): Light yellow solid; Yield 90\%; M.p.: $78-80^{\circ} \mathrm{C} ;{ }^{1} \mathrm{H}$ NMR $\left(300 \mathrm{MHz}, \mathrm{CDCl}_{3}\right): \delta=8.32$ $(d, J=8.8 \mathrm{~Hz}, 2 \mathrm{H}), 7.94(d, J=8.8 \mathrm{~Hz}, 2 \mathrm{H}), 7.29(s$, 1H), 4.32 ( $s, 1 \mathrm{H})$; IR (KBr): 2925, 2852, 2219, 1595,
1510, 1438, 1344, 1258, $1105 \mathrm{~cm}^{-1}$; MS (EI): $m / z 222$

$\left[\mathrm{M}^{+}\right], 187,170,140$ (100), 114; GC: $Z: E=6: 94$.

\section{Results and discussion}

The starting material, Baylis-Hillman adducts are synthesized by modifying the reported procedure by treating various aldehydes with acrylates/acrylonitrile in presence of $30 \mathrm{~mol} \%$ DABCO as a catalyst under solvent-free conditions. In a reference reaction, we have studied the reaction conditions elaborately like selection of base, temperature, and solvent system for the efficacy of the chlorination of Baylis-Hillman adducts. After numerous trails, triphosgene/pyridine/DCM at $0^{\circ} \mathrm{C}$ is found to be the best reaction condition for the effective conversion (scheme 1). Comparative studies of different bases in chlorination of Baylis-Hillman adduct (1m) using triphosgene is tabulated in table 1 . The results of table 1 reveal that aromatic bases like pyridine and DMAP are most effective in this chlorination reaction. The other aliphatic bases viz., DBU, $\mathrm{DABCO}$ and piperidine gives moderate to low yields with more reaction time due to their higher reactivity in the reaction. It was noticed that the reaction was found not progressing using DIPEA and triethylamine. In almost all cases, starting material only recovered from the reaction mixture. Pyridine has chosen as suitable base for the conversion due to its inexpensiveness and with respect to reaction yield/time. The variation of temperature and time does not show any appreciable change in the reaction. To demonstrate the general utility of the reaction, the method was successfully applied to various Baylis-Hillman adducts having both ester (1a-l) and nitrile (1m-v) moieties (table 2). The reaction underwent smoothly in all the cases and excellent

Table 2. Synthesis of allyl chlorides from Baylis-Hillman adducts.

S. No


Table 2. (continued).

4

5

6

7

8<smiles>C=C(C(=O)OC)C(O)c1ccc(OC)cc1</smiles><smiles>C=C(C(=O)OC)C(O)c1ccc(F)cc1</smiles><smiles>C=C(C(=O)OC)C(O)c1ccccc1Cl</smiles>

9

10<smiles>C=C(C(=O)OCC)C(O)c1ccc(Cl)cc1</smiles>

11<smiles>C=C(C(=O)OCC)C(O)c1ccc(OC)cc1</smiles>

12

13<smiles>C=C(C(=O)OCC)C(O)c1ccc(Cl)cc1Cl</smiles><smiles>C=C(C(=O)OCC)C(O)c1ccc([N+](=O)[O-])cc1</smiles><smiles>C=C(C(C)=O)C(O)CC</smiles>

14<smiles>C=C(C#N)C(O)c1ccccc1</smiles>

15<smiles>COC(=O)C(=Cc1ccc(C(C)C)cc1)CCl</smiles>

93 96:4<smiles>COC(=O)C(=Cc1ccc(OC)cc1)CCl</smiles>

90 95:5<smiles>CC(=O)C(=Cc1ccc(F)cc1)CCl</smiles><smiles>COC(=O)/C(=C/c1ccccc1Cl)CCl</smiles><smiles>CCOC(=O)/C(=C/c1ccc(Cl)cc1)CCl</smiles><smiles>CCOC(=O)C(=Cc1ccc(OC)cc1)CCl</smiles><smiles>CCOC(=O)/C(=C/c1ccc(Cl)cc1Cl)CCl</smiles><smiles>CCOC(=O)C(=Cc1ccc([N+](=O)[O-])cc1)CCl</smiles><smiles>CC/C=C(\CCl)C(=O)OC</smiles><smiles>N#C/C(=C\c1ccccc1)CCl</smiles><smiles>CCc1ccc(/C=C(\C)CCl)cc1</smiles>

$2 n$

95<smiles>C/C(=C\c1cccc(F)c1)CCl</smiles> 
Table 2. (continued).

(1)

${ }^{\mathrm{a}} \mathrm{Z} / \mathrm{E}$ ratio was determined by ${ }^{1} \mathrm{H}$ NMR and GC analysis

yields were obtained in short reaction times (immediately). Another importance of the reaction was no column chromatography is required for the isolation of product. Several functionalities such as halogen, nitro, ether, and ester remained intact. The adducts containing electron donating as well as withdrawing groups have been utilized effectively in this protocol and 22 examples are depicted in table 2. See supplementary information.

The stereoselectivity of present conversion was studied in detailed. There are a few reports in the literature regarding stereochemistry of Baylis-Hillman allyl chlorides. ${ }^{7-11,16}$ Following earlier reports, according to ${ }^{1} \mathrm{H}$ NMR data and iterative GC analysis of prepared chlorides, revealed that the stereochemistry of ester containing Baylis-Hillman allyl chlorides major isomer is $Z$ and nitrile containing Baylis-Hillman allyl chlorides major isomer is $E$. The ratio of the $E-Z$ products as measured by GC is listed in the table 2 .

\section{Conclusions}

In summary, we have described a mild and efficient method for the chlorination of Baylis-Hillman adducts using triphosgene. The advantages of triphosgene/pyridine system are; more convenient to handle, operational simplicity, excellent yields, no chromatography, and simple workup procedure. This is a versatile, inexpensive, environmentally benign method and makes this a valid contribution in upgrading to the existing methodologies. Application of this strategy to 
the preparation of new heterocyclic entities from allyl chlorides is currently being pursued.

\section{Supporting information}

The supplementary information for experimental procedure, characteristic data and spectra of new compounds $2 \mathbf{c}, \mathbf{2 d}$ and $\mathbf{2 n}$ can be seen in the website (www. ias.ac.in/chemsci).

\section{Acknowledgements}

The authors thank the Director, Indian Institute of Chemical Technology (IICT) and Head, Organic-II Division-II for encouragement. This work is the Main Lab Project of IICT. TN, CHNSSP and BM thank the University Grants Commission (UGC) and the Council of Scientific and Industrial Research (CSIR), New Delhi for fellowship.

\section{References}

1. (a) Drewes S E and Roos G H P 1988 Tetrahedron 44 4653; (b) Basavaiah D, Dharma Rao P and Suguna Hyma R 1996 Tetrahedron 52 8001; (c) Ciganek E 1997 Organic reactions (ed) L A Paquette (New York: Wiley) vol. 51, p. 201; (d) Ma G-N, Jiang J-J, Shi M and Wei Y 2009 Chem. Commun. 5496

2. (b) Basavaiah D, Reddy B S and Badsara S S 2010 Chem. Rev. 110 5447; (b) Singh V and Batra S 2008 Tetrahedron 64 4511; (c) Basavaiah D, Rao A J and Satyanarayana 2003 Chem. Rev. 103 811; (d) Lee K Y, Gowrisankar S and Kim J N 2005 Bull. Korean Chem. Soc. 26 1481; (e) Radha Krishna P, Sachwani R and Srinivas Reddy P 2008 Synlett. 2897

3. (a) Hoffmann H M R and Rabe J 1984 Helv. Chim. Acta 67 413; (b) Roush Brown B 1993 J. Org. Chem. 58 2151; (c) Mazdiyasani H, Konopacki D B, Dickman D A and Zydowski T M 1993 Tetrahedron Lett. 34 435; (d) Hoffmann H M R and Rabe J 1985 Angew. Chem. Int. Ed. 24 94; (e) Basavaiah D, Bakthadoss $\mathrm{M}$ and Pandiaraju S 1998 Chem. Commun. 1639

4. Börner C, Gimeno J, Gladiali S, Goldsmith P J, Ramazzotti D and Woodward S 2000 Chem. Commun. 2433

5. (a) Das B, Banerjee J, Ravindranath $\mathrm{N}$ and Venkataiah B 2004 Tetrahedron Lett. 45 2425; (b) Radha Krishna P, Kannan V and Sharma G V M 2004 Synth. Commun. 34 55

6. Das B, Shashi Kanth B and Ravinder Reddy K 2008 Chem. Lett. 37512
7. Das B, Chowdhury N, Damodar K and Ravikanth B 2007 Helv. Chim. Acta 902037

8. Li J, Li S, Jia X and Zhang Y 2008 J. Chem. Res. 148

9. (a) Lawrence N J, Crump J P, Mc Gown A T and Hadfield J 2001 Tetrahedron Lett. 42 3939; (b) Biswas K, Börner C, Gimeno J, Goldsmith P J, Ramazzotti D, So A L K and Woodward S 2005 Tetrahedron 61 1433

10. Liu Y, Zheng H, Xu D, Xu Z and Zhang Y 2007 Org. Prep. Proc. Int. 39190

11. Chavan S P, Ethiraj K S and Kamat S K 1997 Tetrahedron Lett. $\mathbf{3 8} 7415$

12. Ying T, Bao W, Wang Z and Zhang Y 2005 J. Chem. Res. Synop. 296

13. (a) Ravi D, Rama Rao N, Reddy G S R, Sucheta K and Jayathirtha Rao V 1994 Synlett. 856; (b) Narendar P, Gangadasu B, Ramesh Ch, China Raju B and Jayathirtha Rao V 2004 Synth. Commun. 34 1097; (c) Ecker H and Forster B 1987 Angew. Chem. Int. Ed. Engl. 26 894; (d) Goren Z, Heeg M J and Mobashery S 1991 J. Org. Chem. 56 7186; (e) Gangadasu B, Chinaraju B and Jayathirtha Rao V 2004 US patent 6737529

14. (a) Gangadasu B, Narender $P$, Bharat Kumar S, Ravinder M, Ananda Rao B, Ramesh Ch, China Raju B and Jayathirtha Rao V 2006 Tetrahedron 62 8398; (b) Cotarca L, Delogu P, Nardelli A and Sunjic V 1996 Synthesis 553, and references therein; (c) Ghosez L, George-Koch I, Patiny L, Houtekie M, Bovy P, Nshimyumukiza P and Phan T 1998 Tetrahedron 54 9207; (d) Flosser D A and Olofson R A 2002 Tetrahedron Lett. 43 4275; (e) Banwell M G, Coster M J, Harvey M J and Moraes J 2003 J. Org. Chem. 68 613; (f) Gumaste V K, Bhawal B M and Deshmukh A R A 2002 Tetrahedron Lett. 43 1345; (g) Kocz R, Roestemadji J and Mobashery S 1994 J. Org. Chem. 59 2913; (h) Peng X, Wang J, Cui J, Zhang R and Yan Y 2002 Synth. Commun. 32 2361; (i) Kluge M and Sicker D 1998 J. Nat. Prod. 61 821; (j) Han K-J and Kim M 2007 Lett. Org. Chem. 4 20; (k) Su W, Weng Y, Zheng C, Zhang Y, Shi F, Hong B, Chen Z, Li J and Li Z 2009 Org. Prep. Proc. Intl. 4193

15. (a) Narender P, Ravinder M, Sadhu P S, China Raju B, Ramesh Ch and Jayathirtha Rao V 2009 Helv. Chim. Acta 92 959; (b) Ravinder M, Sadhu P S and Jayathirtha Rao V 2009 Tetrahedron Lett. 50 4229; (c) Ravinder M, Sadhu P S, Santhoshi A, Narender P, Swamy G Y S K, Ravi Kumar K and Jayathirtha Rao V 2010 Synthesis 573; (d) Narender P, Gangadasu B, Ravinder M, Srinivas U, Swamy G Y S K, Ravikumar K and Jayathirtha Rao V 2006 Tetrahedron 62 954; (e) Kumar Ch N S S P, Parida D K, Santhoshi A, Kota A K, Sridhar B and Jayathirtha Rao V 2011 Med. Chem. Comm. 2486

16. Das B, Majhi A, Banerjee J, Chowdhury $\mathrm{N}$ and Venkateswarlu K 2005 Tetrahedron Lett. 467913 